\title{
A Unique Case of Sclerosing Mediastinal B Cell Lymphoma
}

\author{
Neha Sethi,* Vijay Kaul, C L Pande, Anjali Sharma
}

Dept of Pathology, Bhagwan Mahaveer Cancer Hospital and Research Centre, Jaipur. India

\section{ABSTRACT}

Primary mediastinal B cell lymphoma is a rare subtype of diffuse large B cell Lymphoma (DLBCL) arising from putative thymic B cell origin. It accounts for 2-4 \% of NHL. The present case was a 55 years old male presented with sudden onset of cough. Chest $\mathrm{x}$ ray and CT thorax showed presence of mildly enhancing solid mass in anterior mediastinum. Fine needle aspiration cytology (FNAC) from the mediastinal mass showed presence of poorly differentiated malignant neoplasm. Trucut biopsy from the same showed presence of tumour with foci of hyalinization. On immunohistochemistry tumour cells expressed CD20, CD 30 and CD 23. Hence thediagnosis of NHL of mediastinal sclerosing B cell type was made.

The present case was one of the rare cases of Non Hodgkin Lymphoma (NHL) with an unusual presentation. Primary mediastinal B cell Lymphoma (PMBCL) with sclerosis is a distinct subtype of NHL with unique clinicopathological aspects and aggressive behavior. Prompt recognition and aggressive treatment may lead to relatively longer survival of patients.

\section{Keywords: NHL, Mediastinum, Sclerosing}

\section{Introduction}

Primary mediastinal B cell lymphoma is a rare subtype of DLBCL arising from putative thymic B cell origin. ${ }^{[1]} \mathrm{It}$ accounts for $2-4 \%$ of NHL, occurring predominantly in young adults with female predominance ${ }^{[2]}$ It usually present as a localized mass in anterior mediastinum. Bone marrow and lymph node involvement (other than supraclavicular and cervical) is absent by definition to rule out systemic DLBCL with secondary mediastinal involvement.

\section{Case Report}

A 55 years old male presented with sudden onset of cough with frequent recurrences since 2 months. On investigations, his hemogram was normal. Chest $\mathrm{x}$ ray and CT thorax showed presence of mildly enhancing solid mass in anterior mediastinum of size 110x80x86 mm with presence of necrotic areas which suggested possibility of Lymphoma. There was presence of bilateral pleural effusion. His bone marrow biopsy and bone marrow aspirate showed normocellular marrow. FNAC from the mediastinal mass showed presence of poorly differentiated malignant neoplasm.

Trucut biopsy from the same showed presence of tumor with foci of hyalinization (Fig 1). Tumor cells were medium to large sized with abundant pale cytoplasm and round to ovoid nuclei. Periodic acid Schiff stain showed absence of glycogen. Reticulin stain showed presence of compartmentalising fibrosis (Fig 2a). Hence, a possibility of thymic carcinoma (clear cell type)was given.
On immunohistochemical studies by DAKO-Envision HRP detection system, tumour cells expressed CD20 (Fig 2b), CD 30 and $C D 23$ while immunonegativefor CD3, CK and Vimentin. Hence, diagnosis of NHL of mediastinal sclerosing B cell type was made.

Patient was given treatment following protocol DA-REPOCH + G-CSF. It stands for dose adjusted R-EPOCH that is dose adjusted Rituximab- Etoposide, doxorubicin, cyclophosphamide, vincristine and prednisolone + Granulocytic colony stimulating factors (G-CSF). This therapy obviates the need for radiotherapy in these patients and resulted in event free increased survival rate. ${ }^{[1]}$ The present case responded to treatment and then was discharged with continuous follow up.

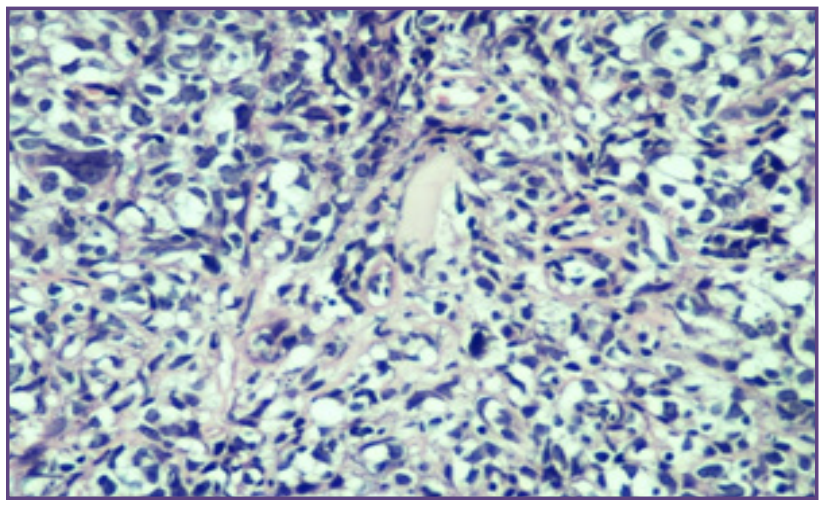

Fig. 1-H/E STAIN (40X) showing tumour with foci of hyalinisation. 


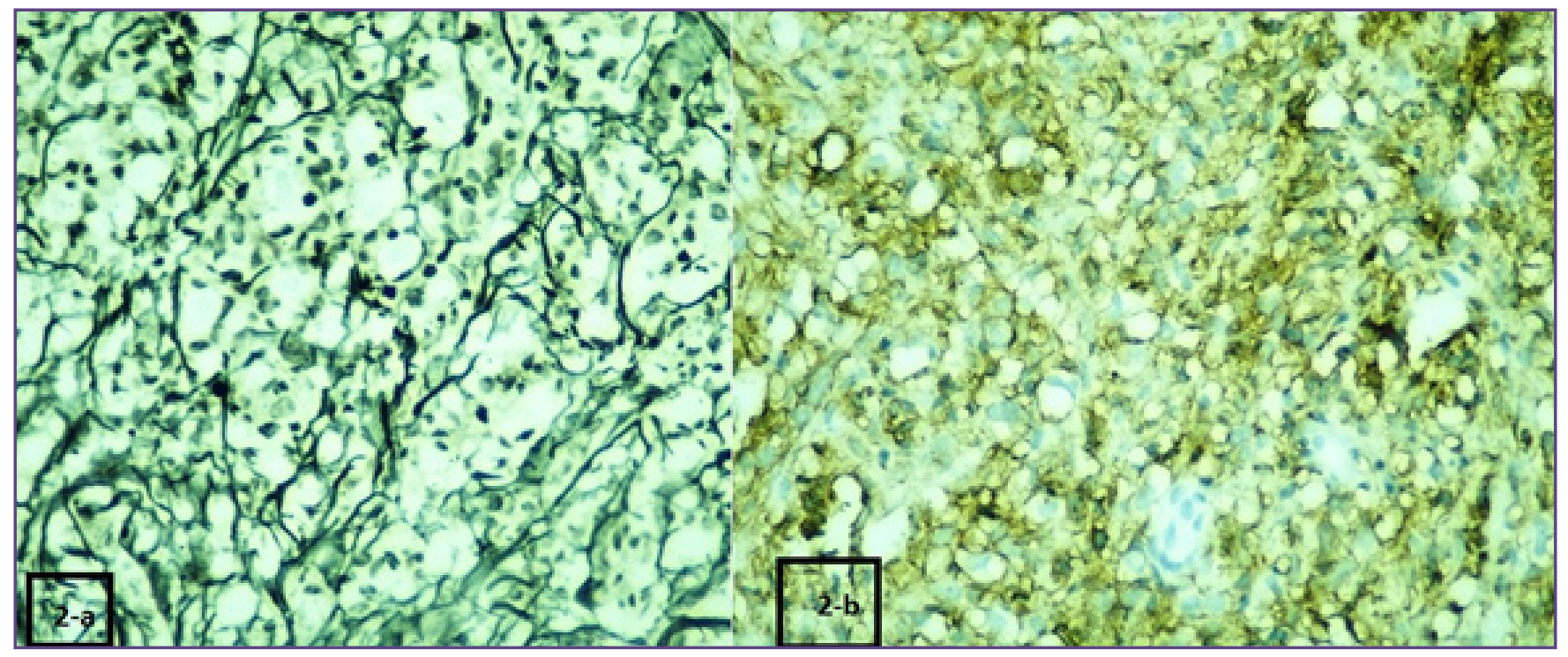

Fig. 2-(a) Reticulin stain showing compartmentalizing fibrosis. (b) IHC (CD20) showing positive tumour cells.

\section{Discussion}

PMBCL was first described by Lichenstein et al in 1980.

${ }^{[2]}$ Thereafter many studies have been done regarding its pathogenesis and treatment and was included in WHO classification.

Primary mediastinal B cell lymphoma is a rare subtype of DLBCL arising from putative thymic B cell origin. ${ }^{[4]}$ It accounts for $2-4 \%$ of NHL, occurring predominantly in young adults with female predominance. ${ }^{[5]}$ It usually present as a localized mass in anterior mediastinum. Bone marrow and lymph node involvement (other than supraclavicular and cervical) is absent by definition to rule out systemic DLBCL with secondary mediastinal involvement.

It has fairly unique clinicopathological presentation. Present case was an adult male which is very unusual presentation. According to Barth et al, ${ }^{[5]}$ elderly patients are more affected by DLBCL as compared to PMBCL which shows involvement of young individuals in $3^{\text {rd }}$ or $4^{\text {th }}$ decade.It is bulky and frequently invades adjacent structures and can involve supraclavicular and cervical lymph node. Bone marrow infiltration is very rare at the time of presentation, as seen in the present case. A study done by Shemmariet al also showed that only $10 \%$ of patients had bone marrow involvement. ${ }^{[6]}$

PMBCL show a wide range of morphology and cytological features depending on individual case. They are usually associated with compartmentalising alveolar fibrosis. ${ }^{[7]}$ The present case also showed sudden onset of cough due to local effects of tumour.

In immunohistochemical studies, tumour cells show positivity for B cell antigens (CD 19, 2022,79 a) but as a rule lack Ig. CD 30, CD 15, MUM -1 and CD 23 are also frequently positive. Recent studies show that most cases of PMBCL also express BOB 1, MAL, CD 54, CD 95 and TRAF $1 .{ }^{[8]}$

In cytogenetic studies, they show gain in chromosome 9 p24 and 2p15. It has unique transcriptional signature, $\mathrm{NF} \mathrm{kb}$ and JAK-STAT signaling pathways. [9] The discordant expression of B cell receptor (CD 79a +, sig -) is characteristic of PMBCL. ${ }^{[10]}$

PMBCL has an equivalent or slightly better prognosis than typical DLBCL. PMBCL should be differentiated from Hodgkins Lymphoma. PMBCL are CD 30 positive but are usually weak and heterogenous compared to HL. Hoeller et al ${ }^{[8]}$ suggest that expression of BOB 1 favours PMBCL and expression of Cyclin E favors CHL.

Some cases show overlap in immunomarkers expression between PMBCL and $\mathrm{HL}$ and are considered to be in grey zone. CD15 is not typically identified in PMBCL and if present should raise the possibility of an overlap diagnosis. ${ }^{[1]]}$

Another important differential diagnosis includes thymic carcinoma in which epithelial component is highlighted by cytokeratin. It should also be differentiated from germinoma as clear cells of PMBCL mimic the germinoma cells. However, absence of glycogen and PAS negativity favours the diagnosis of Lymphoma.

Response to chemotherapy with or without radiotherapy is usually good. Extension into adjacent structures, pleural effusion and male sex has poor prognostic implication as seen in our case. On the other hand, presence of sclerosis denotes good prognosis. ${ }^{[12]}$ 
DA-R-EPOCH treatment protocol is usually given. Some studies show use of MACOP-B (Methotrexate + leucovorin rescue, doxorubicin, cyclophosphamide, vincristine, prednisone and bleomycin) chemotherapy in these patients with favourable outcome. ${ }^{[13]}$

\section{Conclusion}

The present case is one of the rare cases of NHL with an unusual presentation. PMBCL with sclerosis is a distinct subtype of NHL with unique clinic pathological aspects and aggressive behavior. Prompt recognition and aggressive treatment may lead to relatively longer survival of patients.

\section{References}

1. Wilson WH, Grossbard ML, Pittaluga S. Dose adjusted EPOCH chemotherapy for untreated B Cell lymphomas, a pharmacodynamics approach with high efficacy. Blood 2002;99:2685-93.

2. Lichtenstein AK, Levine A, Taylor CR. Primary mediastinal lymphoma in adults. Am J Med. 1980;68(4):509-514.

3. Swerdlow SH, Campo E, Harris NL. WHO classification of tumours of hematopoietic and lymphoid tissue, Lyon, France: IARC Press: 2008: 194-195.

4. Hofmann WJ, Momburg F, Moller P. Thymic medullary cells expressing B lymphocyte antigens. Hum pathol, 1988; 19; 1280-1287.

5. Barth TF, Leithauser F, Joos S, Bentz M, Moller P. Mediastinal (thymic) large B Cell Lymphoma where do we stand? Lancet Oncol, 2002; 3; 229-234.
6. Shemmari SA, Sankarnarayan SP, Krishan Y. Primary Mediastinal large cell lymphoma. Clinical features, prognostic factors and survival with RCHOP in Arab patients in the PET Scan era. Lung India. 2014 Jul-Sep; 31(3):228-231.

7. Paulli M, Strater J, Gianelli U, Rousset MT, Gambacorta M, Orlandi E, et al. Mediastinal B cell lymphoma, a study of its histomorphological spectrum based on 109 cases. Hum pathol, 1999; 30; 178-187.

8. Hoeller S, Zihler D, Zlobec I. BOB 1, CD79a, Cyclin E are the most appropriate markers to discriminate classical Hodkins Lymphoma from PMLBCL. Histopathology. 2010; 56(2):217-228.

9. Melzner I, Bucur AJ, Bruderlein S, Dorsch K, Hasel C, Barth F. Biallelic mutation of SOCS-1 impairs JAK 2 degradation and sustains phosphor -JAK 2 action in the MedB-1 mediastinal Lymphoma line. Blood. 2005: 105:2535-2542.

10. Kanavaros P, Gaulard P, Charlotte F, Martin N, Ducos C, Lebezu M, et al. Discordant expression of immunoglobulin and its associated molecule mb -1/CD 79a is frequently found in mediastinal large Bcell lymphomas. Am J Pathol. 1995; 146:735-741.

11. Hoeller S, Bergman CC. Grey zone Lymphoma: Lymphoma with intermediate features. AdvHematol 2012; Article id 460801, 7 pages doi:10.1155/2012/460801.

12. Johnson PW, Davies AJ. Primary mediastinal B Cell Lymphoma. Hematology Am Soc; 2008; 349-358.

13. Klimo P, Connors JM. MACOP-B chemotherapy for the treatment of diffuse large cell lymphoma.Ann Intern Med 1985 May; 102(5):596-602.

*Corresponding author:

Dr. Neha Sethi, Dept of Pathology, Bhagwan Mahaveer Cancer Hospital and Research Centre, Jaipur. India

Phone: +91 9983947655.

Email: nehasethi3@gmail.com

Financial or other Competing Interests: None. 